\title{
Use of medications by people with chronic fatigue syndrome and healthy persons: a population-based study of fatiguing illness in Georgia
}

\author{
Roumiana S Boneva*, Jin-Mann S Lin, Elizabeth M Maloney, James F Jones \\ and William C Reeves
}

Address: Centers for Disease Control and Prevention, 1600 Clifton Road, Atlanta, Georgia 30333, USA

Email: Roumiana S Boneva* - rboneva@cdc.gov; Jin-Mann S Lin - jlin2@cdc.gov; Elizabeth M Maloney - emaloney1@cdc.gov; James F Jones - jfjones@cdc.gov; William C Reeves - wreeves@cdc.gov

* Corresponding author

This article is available from: http://www.hqlo.com/content/7/l/67

(C) 2009 Boneva et al; licensee BioMed Central Ltd.

This is an Open Access article distributed under the terms of the Creative Commons Attribution License (http://creativecommons.org/licenses/by/2.0), which permits unrestricted use, distribution, and reproduction in any medium, provided the original work is properly cited.

\begin{abstract}
Background: Chronic fatigue syndrome (CFS) is a debilitating condition of unknown etiology and no definitive pharmacotherapy. Patients are usually prescribed symptomatic treatment or selfmedicate. We evaluated prescription and non-prescription drug use among persons with CFS in Georgia and compared it to that in non-fatigued Well controls and also to chronically Unwell individuals not fully meeting criteria for CFS.

Methods: A population-based, case-control study. To identify persons with possible CFS-like illness and controls, we conducted a random-digit dialing telephone screening of 19,807 Georgia residents, followed by a detailed telephone interview of 5,630 to identify subjects with CFS-like illness, other chronically Unwell, and Well subjects. All those with CFS-like illness $(n=469)$, a random sample of chronically Unwell subjects $(n=505)$, and Well individuals $(n=64 I)$ who were age-, sex-, race-, and geographically matched to those with CFS-like illness were invited for a clinical evaluation and 783 participated (48\% overall response rate). Clinical evaluation identified II3 persons with CFS, 264 Unwell subjects with insufficient symptoms for CFS (named ISF), and I24 Well controls; the remaining 280 subjects had exclusionary medical or psychiatric conditions, and 2 subjects could not be classified. Subjects were asked to bring all medications taken in the past 2 weeks to the clinic where a research nurse viewed and recorded the name and the dose of each medication.
\end{abstract}

Results: More than $90 \%$ of persons with CFS used at least one drug or supplement within the preceding two weeks. Among users, people with CFS used an average of 5.8 drugs or supplements, compared to $4 . I$ by ISF and 3.7 by Well controls. Persons with CFS were significantly more likely to use antidepressants, sedatives, muscle relaxants, and anti-acids than either Well controls or the ISF group. In addition, persons with CFS were significantly more likely to use pain-relievers, antihistamines and cold/sinus medications than were Well controls.

Conclusion: Medical care providers of patients with chronic fatigue syndrome should be aware of polypharmacy as a problem in such patients, and the related potential iatrogenic effects and drug interactions. 


\section{Background}

Chronic fatigue syndrome (CFS) is diagnosed based on self-reported symptoms and exclusion of other illnesses that could cause the symptoms. There are no diagnostic clinical signs or laboratory markers for CFS. Thus, both health care providers and patients express concern about uncertainties in the diagnosis and management of the illness. This may be reflected in the apparent conundrum that persons with CFS have on average 22 healthcare visits per year [ 1 ] while only $20 \%$ of persons with CFS identified from the general population have been diagnosed with CFS $[2,3]$.

Because the cause and pathogenesis of CFS remain inchoate, no definitive pharmacotherapy exists [4]. Many health care providers prescribe medications to treat the most bothersome symptoms - fatigue, muscle or joint pain, un-refreshing sleep and cognitive impairment. Most people with CFS who are under medical care have been ill for at least 5-years and may become frustrated with a lack of acceptable recovery. They often consult several providers and also self-medicate to treat their symptoms $[5,6]$. However, both prescribed and over the counter medications may cause untoward side effects, which may lead to new symptoms and exacerbate overall disability. We are aware of only one published population-based study (conducted in Wichita, Kansas) that documented medication use by persons suffering CFS and found that persons with CFS were more likely to use pain relievers, hormones, antidepressants, gastrointestinal and central nervous system medications [7]. We conducted the present analysis to critically evaluate use of prescription and nonprescription drugs (and supplements) by persons with CFS as compared to Well controls and persons who do not fully meet criteria for CFS (referred to as ISF). We used more recent data collected from defined metropolitan, urban, and rural populations in Georgia.

\section{Methods}

\section{Study design}

The study was approved by the Institutional Review Board of the Centers for Disease Control and Prevention and adhered to the human research guidelines of the U.S. Department of Health and Human Services. All participants were volunteers who gave informed consent.

We conducted a population-based, case-control study to identify persons with CFS, Unwell and Well persons. Figure 1 represents a flow chart of how the subject sample was derived and details have been published earlier [8]. Briefly, between September 2004 and July 2005 we used random digit dialing to conduct a household screening interview with a household informant in three geographic areas in Georgia (metropolitan, urban and rural). The household informant described demographics and health status of household members 18 to 59 years old; that initial interview enumerated 19,807 adult residents and screened for unwellness among household members, based on having at least one CFS symptom (fatigue, impaired cognition, un-refreshing sleep, muscle or joint pain); Well residents had none of these symptoms for $\geq 1$ month. The screening interview revealed 10,834 (55\%) Well persons, 5,122 (26\%) persons who were Unwell for at least a month but not fatigued, and 3,851 (19\%) who were Unwell and fatigued for at least a month. We then conducted detailed telephone interviews with all those identified as Unwell with fatigue, a random selection of those who were Unwell but without fatigue and a random sample of Well persons (see Figure 1). Based on their responses to the detailed telephone interview, we classified participants as CFS-like if they met criteria of the 1994 CFS case definition [9]; as chronically Unwell if they endorsed some but not all CFS symptoms and as Well if they reported no such symptoms. Finally, we invited all 469 persons classified as CFS-like, 641 Well persons matched to the CFS-like by sex, race/ethnicity, age, and geographic stratum and a similar number $(n=505)$ of randomly selected Unwell persons for a one day clinical evaluation. Overall, $48.5 \%$ completed the clinical evaluation.

\section{IIIness classification}

To identify medical conditions considered exclusionary for CFS $[9,10]$, the clinical evaluation included a standardized past medical history, a review of systems, a standardized physical examination, and routine laboratory testing of blood and urine. To identify psychiatric conditions considered exclusionary for CFS, licensed and specifically trained psychiatric interviewers administered the Structured Clinical Interview for DSM-IV (SCID) to diagnose Axis I psychiatric disorders and the Zung self-rating depression scale (SDS) to measure severity of depression [11]. Medical and psychiatric evaluations identified medical or psychiatric conditions considered exclusionary for CFS in 280 (36\%) of the clinic participants; they and two others who had incomplete data were excluded from the analyses, leaving a total sample of 501 subjects for analyses.

We diagnosed CFS according to criteria of the 1994 case definition [9] and as recommended by the International CFS Study Group [10], which is standard in CDC studies of CFS $[8,12]$. Thus, we evaluated functional impairment by means of the Medical Outcomes Short-Form Health Survey (SF-36) [13]; we used the Multidimensional Fatigue Inventory (MFI-20) [14] to measure characteristics of fatigue and we utilized the CDC CFS Symptom Inventory to document occurrence, frequency and severity of the defining symptoms [15]. Subjects who had $\geq 4$ case defining symptoms and exceeded the Symptom Inventory 

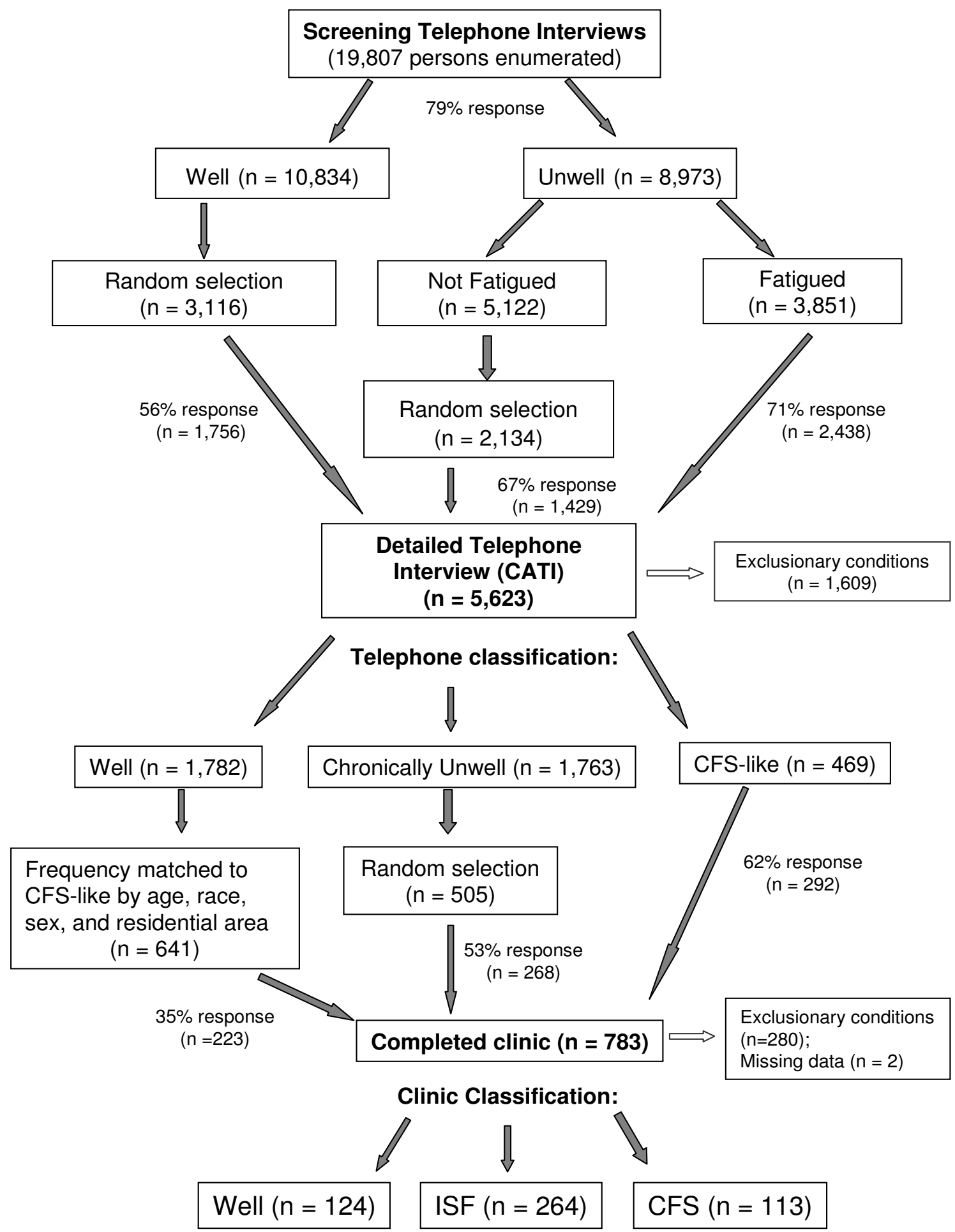

Figure I

Flow chart of subject sample derivation for the population-based, case-control study of chronic fatigue syndrome in Georgia, USA, 2004-2005. 
cut-off score, and met CFS cut-off scores on the SF-36 and the MFI-20, were considered to have CFS ( $n=113$ participants). Those who met at least one, but not all CFS criteria, comprised the ISF group $(n=264)$ and those who met none of the cut-off criteria comprised the Well group $(\mathrm{n}=$ 124).

\section{Data collection}

We solicited demographic information during the detailed telephone interview and confirmed it at clinic. Clinic participants completed a battery of questionnaires prior to their clinic appointment, including questions concerning annual household income and health care utilization. In addition to completing questionnaires, we instructed participants to bring all medications (prescription and over the counter drugs and supplements) used within the past 2 weeks to their clinic appointment, where a nurse recorded the name, dose, reason and frequency of use. Information on reason for taking a medication was obtained primarily by general inquiry and recorded by clinical investigators using participant's or investigator's terminology of their own choosing.

For the purpose of this study we use the term "drugs" to refer to all prescription medicines and all non-prescription medicines that are available over the counter, but are not supplements or homeopathic medications. We use the term "supplements" to denote nutritional supplements, including vitamins, minerals, amino acids, fatty acids, homeopathic preparations and herbs.

A physician review panel from the CDC CFS Research Program reviewed the verbatim data recorded at clinic and verified names of drugs and supplements by means of the Physicians Desk Reference (PDR) or through website databases. The panel utilized generic name and ingredients to categorize individual drugs into 287 groups and an additional group for supplements. Based on their main effects, we grouped drugs into a smaller number of major categories. For the purpose of this study we kept the major drug categories similar to our previous study of drug use by persons with CFS [7]. The present analysis is limited to drugs used by at least 5 of the 501 subjects.

\section{Statistics}

We used Chi-square $\left(\chi^{2}\right)$ or Fisher's exact tests of independence to compare the distribution of categorical demographic characteristics by the three study groups and to assess differences in frequency of use of various medications by the three study groups. We used the KruskalWallis test to compare differences in income, age and BMI by study groups. We used logistic regression to compute odds ratios (OR) for medication use in the CFS group relative to the ISF and Well groups; the Wald test was used to compute $95 \%$ confidence intervals as measures of the precision of the OR. We adjusted the analyses for potential confounders (household income, BMI, age, sex, race and geographic stratum) by including them as covariates in the regression models. The Hosmer-Lemeshow test served to assess the goodness of fit for multivariate logistic regression models.

\section{Results \\ Descriptives and demographics}

The CFS group was similar to the ISF and Well groups with respect to the distribution of age, sex, race and geographic stratum (Table 1). The Well group had a significantly higher household income $(\mathrm{p}<0.001)$ and significantly lower BMI compared to the CFS and ISF groups $(\mathrm{p}<0.01$ for both).

\section{Overall use of drugs and supplements}

The 501 participants brought in 2,205 individual preparations that they were taking, of which we considered 1,557 to be drugs and 648 to be supplements (as defined above). Virtually every clinic participant (95.6\% of the CFS; $88.6 \%$ of the ISF; and $90.3 \%$ of the Well) brought in a drug or supplement they had taken over the last two weeks (table 2). The average number of preparations (drugs or supplements) used was 5.8 in the CFS group (median 4, range 1-29), 4.1 in the ISF group (median 3.0, range $1-20$ ), and 3.7 in the Well group (median 3, range $1-18$ ). Overall, $85.8 \%$ of the entire sample (430 of 501) used at least one drug: $92.9 \%$ of CFS, $83.7 \%$ of ISF and $83.9 \%$ of the Well group. The mean number of drugs used per person in the CFS group was 4.3 (median 3, range 119); in the ISF group it was 3.0 (median 2, range 1-12), and in the Well group it was 2.9 (median 2, range 1-15). In contrast to drugs, the prevalence of supplement use was lower in the CFS (44.2\%) and the ISF (44.3\%) groups compared to the Well group (52.4\%).

\section{Use of specific medication categories}

Overall, in the combined sample $(\mathrm{n}=501)$, the most frequently used categories were pain relievers (55.1\%), supplements (43.1\%), cold/sinus drugs (34.9\%) and antiallergy drugs $(34.9 \%)$ (both latter groups largely represented by antihistamines - 28.1\%), female hormonal drugs (26.7\% of all women), antidepressants $(20.0 \%)$ and anti-acid drugs (16.8\%). Table 2 provides details of frequency of use by drug category for each study group. Table 3 summarizes the results of multivariate logistic regression models predicting drug and supplement use by study groups adjusted for age, BMI, income, sex, race, and geographic area (for a detailed version of this table see Additional file 1). Compared to both the Well controls and the ISF group, the CFS group was significantly more likely to use pain relievers (all and narcotic), antidepres- 
Table I: Basic demographic characteristics of the subjects with chronic fatigue syndrome (CFS), subjects with insufficient symptoms to be CFS (ISF) and Well controls

\begin{tabular}{|c|c|c|c|c|}
\hline Demographic characteristic & $\begin{array}{r}\text { CFS } \\
(n=113)\end{array}$ & $\begin{array}{r}\text { ISF } \\
(n=264)\end{array}$ & $\begin{array}{r}\text { Well } \\
(n=124)\end{array}$ & $P$ \\
\hline Race, n (\%) & & & & 0.19 \\
\hline Caucasian & $84(74.3)$ & $196(74.2)$ & $95(76.6)$ & \\
\hline Black & $21(18.6)$ & $55(20.8)$ & $28(22.6)$ & \\
\hline All other & $8(7.1)$ & $13(4.9)$ & $\mathrm{I}(0.8 \mathrm{I})$ & \\
\hline Area, n (\%) & & & & 0.98 \\
\hline Metro & $23(20.4)$ & $54(20.5)$ & $22(17.7)$ & \\
\hline Urban & $37(32.7)$ & $84(31.8)$ & $42(33.9)$ & \\
\hline Rural & $53(46.9)$ & $126(47.7)$ & $60(48.4)$ & \\
\hline Female sex, n (\%) & $92(81.4)$ & $201(76.1)$ & $93(75.0)$ & 0.44 \\
\hline Age in years, mean (sd) & $44.3(10.1)$ & $43.1(10.4)$ & $44.5(10.5)$ & 0.37 \\
\hline Median age & 44.0 & 45.0 & 47.0 & \\
\hline Age range & $18-59$ & $18-59$ & $19-59$ & \\
\hline BMI, mean (sd) & $27.5(5.0)$ & $27.5(5.2)$ & $26.0(5.3)$ & 0.018 \\
\hline Median BMI & 27.0 & 27.0 & 25.0 & \\
\hline BMI range & $17-39$ & $16-39$ & $18-38$ & \\
\hline Income & & & & 0.017 \\
\hline Mean (sd) & $64,495.8(87,057.0)$ & $67,455.6(63,118.1)$ & $85,599.2(82,699.2)$ & \\
\hline Median & $52,025.0$ & $55,000.0$ & $72,272.0$ & \\
\hline Income range & $0.0-750,000.0$ & $0.0-447,466.0$ & $0.0-500,000.0$ & \\
\hline
\end{tabular}

BMI, Body mass index.

sants, acid-reducing gastro-intestinal medications, sedatives (largely benzodiazepines), and muscle relaxants. Compared to the Well (but not the ISF) group, the CFS group was also more likely to be taking non-steroid antiinflammatory drugs, NSAIDs, (when aspirin was excluded) and anti-allergy drugs and cold/sinus (mostly anti-histamines), and less likely to be taking aspirin. In addition, compared to the ISF group, the CFS group was more likely to be taking thyroid hormone replacement and anti-migraine drugs (all p < 0.05). We further examined those drug categories that were significantly more frequently used by the CFS group and we present the results in descending order of frequency of use.

\section{Pain relievers}

Pain-relievers were the most commonly used drugs in all three groups and the CFS group (65.5\% use) was significantly more likely than the ISF $(51.5 \%)$ or the Well group $(53.2 \%)$ to use pain relievers (including NSAIDs and narcotic medications) (tables 2 and 3). Among users of NSAIDs, bodily pain was the most frequently reported reason for use in all diagnostic groups: $62.2 \%$ of the CFS group, $47.6 \%$ of the ISF group and $52.9 \%$ of the Well group. Arthritis was reported as a reason significantly more frequently in the CFS group compared to the ISF group and the Well group $(28.9 \%, 12.2 \%$ and $5.9 \%$, respectively, $\mathrm{p}=0.004$ for linear trend, $\mathrm{p}=0.01$ for CFS vs. Well). Headache was the second most commonly reported reason for taking NSAIDs among the ISF and Well groups (37\% and $35.3 \%$, respectively), but the third most frequently reported reason $(22.2 \%)$ in the CFS group.
The profile of NSAID use differed between persons with CFS and Well controls. Among persons taking NSAIDs, $49.1 \%$ of the users in the CFS group used ibuprofen compared to the $37.7 \%$ of users in the Well group while, conversely, acetylsalicylic acid (aspirin) was used less frequently in the CFS group $(28.3 \%)$ and the ISF group $(32.7 \%)$ than the Well group (where virtually half (49.1\%) of all NSAID use was accounted for by aspirin). Similarly, overall use of aspirin was lower in the CFS and ISF groups (13.3\% of all subjects in each group) compared to the Well group (21.8\% of subjects). Thus, persons with CFS were 32\% less likely than Well controls to be taking aspirin $\left(\mathrm{OR}_{\mathrm{adj}}=0.68,95 \% \mathrm{CI}, 0.47-0.99, \mathrm{p}=\right.$ $0.049)$. Of the entire Well group, $11.3 \%$ reported preventive use of aspirin (for "heart health/prevention") versus only $6.2 \%$ of the entire CFS group $(p=0.17)$ and $5.7 \%$ of the ISF group $(\mathrm{p}=0.05)$. Other reported reasons for using aspirin were mainly headache or bodily pain, with similar proportions in the three groups (5.3\% of CFS, $6.8 \%$ of ISF and $8 \%$ of $W e l l$ ). After excluding aspirin from the NSAID category the difference in NSAID use between the CFS group and Well controls was significant $(\mathrm{p}=0.03$, table 3$)$.

Acetaminophen-containing drugs were used significantly more frequently by the CFS group (23.9\%) compared to $14.4 \%$ of the ISF group and $11.3 \%$ of the Well controls (tables 2 and 3). The major reported reason (over 55\%) in all groups was headache. However, $37 \%$ of the CFS group used such drugs also to treat bodily pain, versus only $13.2 \%$ of ISF and $7.1 \%$ of Well controls. 
Table 2: Categories of medications used by subjects with chronic fatigue syndrome (CFS), insufficient symptoms/fatigue (ISF) and Well controls in Georgia

\begin{tabular}{|c|c|c|c|c|c|}
\hline \multirow[t]{2}{*}{ Drug category } & CFS & ISF & Well & \multicolumn{2}{|c|}{ p-value } \\
\hline & \multicolumn{3}{|c|}{$N(\%)$ users } & CFS vs. Well & CFS vs. ISF \\
\hline Pain relievers (includes all NSAIDs and narcotics) & $74(65.5)$ & $136(5 \mid .5)$ & $66(53.2)$ & 0.056 & 0.02 \\
\hline -NSAIDs (aspirin included) & $53(46.9)$ & $107(40.5)$ & $55(44.4)$ & 0.74 & 0.31 \\
\hline -NSAIDs (aspirin excluded) & $45(39.8)$ & $82(31.1)$ & $34(27.4)$ & 0.043 & 0.10 \\
\hline -Acetaminophen-containing & $27(23.9)$ & $38(14.4)$ & $14(11.3)$ & 0.011 & 0.026 \\
\hline -Narcotic pain relievers & $19(16.8)$ & $16(6.1)$ & $5(4.0)$ & 0.001 & 0.001 \\
\hline -Aspirin containing & $15(13.3)$ & $35(13.3)$ & $27(21.8)$ & 0.09 & 1.00 \\
\hline Supplements/vitamins & $50(44.2)$ & $117(44.3)$ & $65(52.4)$ & 0.158 & 0.68 \\
\hline Anti-allergy medications (anti-histamines, nasal steroids, sympathomimetics) & $46(40.7)$ & $94(35.6)$ & $35(28.2)$ & 0.04 & 0.36 \\
\hline Asthma medications & $9(7.96)$ & $9(3.4)$ & $3(2.4)$ & 0.097 & 0.065 \\
\hline Cold/sinus medications & $46(40.7)$ & $95(36.0)$ & $34(27.4)$ & 0.025 & 0.4 \\
\hline Anti-histamines & $40(35.4)$ & $74(28.0)$ & $27(21.8)$ & 0.017 & 0.17 \\
\hline Antidepressants & $41(36.3)$ & $48(18.2)$ & $11(8.9)$ & $<0.0001$ & 0.0007 \\
\hline Female hormones & $28(30.4)$ & $49(24.4)$ & $26(28.0)$ & 0.68 & 0.3 \\
\hline - Birth control & $6(6.5)$ & $20(9.9)$ & $\mathrm{II}(\mathrm{II} .8 \%)$ & 0.11 & 0.34 \\
\hline - Hormone replacement & $19(20.7)$ & $28(13.9)$ & $13(14 \%)$ & 0.23 & 0.15 \\
\hline Gastrointestinal, acid-reducing drugs & $30(26.6)$ & $38(14.4)$ & $16(12.9)$ & 0.0082 & 0.009 \\
\hline All cardiovascular & $21(18.6)$ & $46(17.4)$ & $26(21.0)$ & 0.90 & 0.86 \\
\hline Sedatives (including benzodiazepines) & $20(17.7)$ & $18(6.8)$ & $5(4.0)$ & 0.002 & 0.004 \\
\hline - Benzodiazepines only & $14(12.4)$ & $14(5.3)$ & $3(2.4)$ & 0.003 & 0.027 \\
\hline Lipid-lowering & $13(11.5)$ & $31(11.7)$ & $13(10.5)$ & 0.69 & 0.56 \\
\hline Thyroid hormones & $12(10.6)$ & II (4.2) & $8(6.5)$ & 0.28 & 0.04 \\
\hline Muscle relaxants & $10(8.9)$ & $8(3.0)$ & 0 & $<0.001$ & 0.002 \\
\hline Antibiotics & $8(7.1)$ & $19(7.2)$ & $6(4.8)$ & 0.53 & 0.82 \\
\hline Anti-migraine & $7(6.2)$ & $5(1.9)$ & $4(3.2)$ & 0.47 & 0.047 \\
\hline Amphetamines & $5(4.4)$ & $7(2.65)$ & $2(1.6)$ & 0.20 & 0.37 \\
\hline Glucose-lowering & I (0.9) & $10(3.8)$ & $4(3.2)$ & 0.51 & 0.13 \\
\hline Any category & $108(95.6)$ & $234(88.6)$ & $112(90.3)$ & 0.12 & 0.03 \\
\hline
\end{tabular}

NSAID, Nonsteroid anti-inflammatory drug, HRT, hormone replacement therapy

a Percentages for female hormones are calculated for $n=386$ women $(n=92$ CFS, $n=20$ I ISF, and $n=93$ Well $)$

Use of anti-migraine drugs was significantly associated with CFS when compared to the ISF group $\left(\mathrm{OR}_{\mathrm{adj}}=3.44\right.$, $95 \% \mathrm{CI}=1.06,11.10, \mathrm{p}=0.04)$ but not when compared to the Well controls (tables 2 and 3 ).

Persons with CFS were significantly more likely than Well controls (ORadj. $=2.24 ; 95 \% \mathrm{CI}, 1.32-8.8$ ) or the ISF group $(\mathrm{OR}=3.23 ; 95 \% \mathrm{CI}, 1.55-6.75)$ to use narcotic pain relievers. Users of narcotic pain relievers reported neck and back pain as the most frequent reasons $(42.1 \%$ of the users in the CFS group, $37.6 \%$ in the ISF, and $40 \%$ in the Well group). Other reported reasons were pain in the extremities and headache/migraine. Almost half $(47.4 \%)$ of the users of narcotic pain relievers in the CFS group and $18.8 \%$ of the users in the ISF group reported just pain, without specifying its localization, as a reason.

\section{Antihistamines}

Persons with CFS were significantly more likely than Well controls $(p=0.013)$ or the ISF group $(p=0.085)$ to use antihistamines, which comprised the vast majority of anti-allergy and "cold/sinus" drugs (see tables 2 and 3). Major reported reasons for using anti-histamines were allergies or colds/sinus problems $(80 \%$ of antihistamine users in the CFS group, $77 \%$ in the ISF group and $82.6 \%$ in the Well group). Using antihistamines as a sleep aid was almost twice as common in the CFS group (20.0\%) and the ISF group $(20.3 \%)$ compared to the Well group $(11.1 \%)$.

\section{Antidepressants}

A significantly higher proportion of persons with CFS (36.3\%) used antidepressants compared to Well controls $(8.9 \%)$ and persons with ISF $(18.2 \%)(\mathrm{p}<0.001$ for both, see tables 2 and 3 ). Among users of antidepressants, the most commonly reported reason was depression $(64.8 \%$, overall or $63.4 \%$ of the CFS group, $58.3 \%$ of the ISF group and $72.7 \%$ of the Well group). Other reported reasons included anxiety (or "nerves") in $24.3 \%$ of the CFS group, $20.9 \%$ of the ISF group, and $9.1 \%$ of the Well group, and 
Table 3: Adjusted odds ratios for associations between illness status and use of specific drug categories or supplements

\begin{tabular}{|c|c|c|c|c|}
\hline \multirow[t]{2}{*}{ Drug category } & \multicolumn{2}{|c|}{ CFS versus Well } & \multicolumn{2}{|c|}{ CFS versus ISF } \\
\hline & OR $(95 \% \mathrm{Cl})^{\mathrm{a}}$ & $P$ value & OR $(95 \% \mathrm{Cl})$ & $P$ value \\
\hline Muscle relaxants & undefined & 0.000 & $2.76(1.02-7.43)$ & 0.045 \\
\hline Sedatives & $2.49(1.47-4.21)$ & 0.0007 & $3.01(1.49-6.11)$ & 0.002 \\
\hline - Benzodiazepines & $2.49(1.29-4.80)$ & 0.006 & $2.70(1.22-6.00)$ & 0.015 \\
\hline Antidepressants & $2.47(1.68-3.64)$ & $<0.0001$ & $2.40(1.42-4.04)$ & $<0.0001$ \\
\hline Asthma medications & $1.86(0.94-3.67)$ & 0.074 & $2.47(0.94-6.47)$ & 0.065 \\
\hline Anti-histamines & $1.49(1.09-2.03)$ & 0.013 & $1.53(0.94-2.50)$ & 0.085 \\
\hline Cold/sinus & $1.44(1.07-1.93)$ & 0.015 & $1.29(0.80-2.08)$ & 0.29 \\
\hline Anti-migraine & $1.43(0.75-2.73)$ & 0.28 & $3.44(1.06-11.10)$ & 0.039 \\
\hline Anti-allergy & $1.40(1.05-1.88)$ & 0.024 & $1.32(0.82-2.13)$ & 0.25 \\
\hline $\begin{array}{l}\text { Pain relievers } \\
\text { (includes NSAIDs and narcotics) }\end{array}$ & $1.33(1.00-1.77)$ & 0.049 & $1.93(1.20-3.11)$ & 0.007 \\
\hline - Narcotic pain relievers & $2.24(1.32-3.80)$ & 0.003 & $3.23(1.55-6.75)$ & 0.002 \\
\hline - Acetaminophen & $1.68(1.15-2.45)$ & 0.007 & $0.52(0.29-0.91)$ & 0.02 \\
\hline -NSAIDs (aspirin excluded) & $1.38(1.02-1.85)$ & 0.03 & $1.54(0.96-2.48)$ & 0.07 \\
\hline -NSAIDs (aspirin included) & $1.05(0.80-1.39)$ & 0.71 & $1.35(0.85-2.15)$ & 0.20 \\
\hline - Aspirin (alone) & $0.68(0.47-0.99)$ & 0.049 & $0.99(0.47-2.06)$ & 0.97 \\
\hline Gastrointestinal (all acid-reducing drugs) & $1.67(1.17-2.38)$ & 0.005 & $2.17(1.24-3.80)$ & 0.007 \\
\hline Thyroid hormones (all, $3 \mathrm{I} / 50 \mathrm{I}$ ) & $1.32(0.79-2.18)$ & 0.28 & $2.60(1.03-6.57)$ & 0.043 \\
\hline Antibiotics & $1.26(0.7 I-2.21)$ & 0.43 & $0.88(0.36-2.15)$ & 0.79 \\
\hline Supplements & $0.88(0.66-1.17)$ & 0.37 & $0.98(0.61-1.58)$ & 0.93 \\
\hline Cardiovascular drugs & $0.86(0.60-1.24)$ & 0.42 & $1.08(0.58-2.03)$ & 0.81 \\
\hline Glucose-lowering (insulin and oral) & $0.53(0.08-1.71)$ & 0.46 & $0.23(0.03-1.80)$ & 0.16 \\
\hline
\end{tabular}

a, $\mathrm{Cl}$, confidence interval. Odds ratios were adjusted for confounding factors (age, BMI, household income) and sex and geographic area, if indicated. Note. Results are arranged in descending order of odds ratios for use of major drug categories by the CFS group vs. the Well group. Right justified in the first column are drugs (individual drugs or sub-categories) from the preceding major drug category above (left justified). Supplements are included in the table for completeness.

Values of the Hosmer-Lemeshow goodness of fit test ranged from 0.16 to 0.97 (values greater than $>0.05$ reflect good model fit, higher values reflect better fit); individual values are presented in a detailed version of this table available as an additional file.

sleep problems $(14.6 \%, 4.2 \%$ and $9.1 \%$ of the CFS, ISF and Well groups, respectively). Using an SDS score of 50 or higher to indicate depression [11], CFS subjects had the highest SDS index scores $(56.2 \pm 0.9$, mean \pm SEM) followed by the ISF group $(50.3 \pm 0.5)$ and the Well controls $(36.3 \pm 0.4)$. Within each group, the mean SDS index of persons taking antidepressants was similar to the SDS index of those not taking antidepressants: CFS: $56.2 \pm 1.4$ vs. $56.1 \pm 1.5$, respectively; ISF: $50.3 \pm 1.2$ vs. $46.0 \pm 0.7$, respectively; and Well controls: $36.3 \pm 1.4$ vs. $36.5 \pm 0.6$, respectively. The average doses of antidepressants (expressed for each antidepressant as percent of usual adult dose as recommended by PDR) were $142.1 \pm 11.1 \%$ (mean \pm SEM) in the CFS group and $119.6 \pm 16.7 \%$ in the Well group, suggesting that the higher SDS scores in persons with CFS receiving antidepressants could not be accounted for by prescription of lower doses of antidepressants than in the control group.

\section{Gastrointestinal drugs (simple acid reducers, $\mathrm{H} 2$ blockers and proton pump inhibitors)}

Persons with CFS were significantly more likely than the Well controls $(p=0.005)$ or the ISF group $(p=0.007)$ to use acid-reducing gastrointestinal drugs (table 3). Across the groups, the major reason for anti-acid medication use was acid reflux/heartburn, which was reported by $73.4 \%$, followed by "gas or indigestion" (15.1\%). Two persons with CFS (6.7\%) and 2 persons in the ISF (5.3\%) reported ulcer or gastritis as a reason for use. One person with CFS reported specifically that they were taking such drugs to reduce the stomach side effects of an NSAID (etodolac). Among users of pain-relieving/anti-inflammatory drugs only, concurrent use of anti-acid drugs was significantly more common in the CFS group - 27.0\% (20 of 74) than in the ISF group $-17.7 \%$ (24 of 136) or the Well group $12.1 \%$ ( 8 of 66 ), $\mathrm{p}$ for linear trend $=0.02$. Similarly, in the entire sample, concurrent use of anti-acid drugs and painrelieving/anti-inflammatory drugs occurred significantly more frequently in the CFS group - 17.7\%, (20 of 113), than in the ISF group $9.1 \%$, (24 of 264) or the Well group $6.5 \%$ ( 8 of 124), $\mathrm{p}$ for linear trend $=0.005$.

\section{Sedatives}

Persons with CFS were also significantly more likely than Well controls $(\mathrm{p}=0.0007)$ or the ISF group $(\mathrm{p}=0.002)$ to use sedatives, largely accounted for by benzodiazepines (see tables 2 and 3). Reported indications were similar among users for all three groups and included: sleep prob- 
lems in $42.9 \%, 50 \%$ and $40 \%$, for the CFS, ISF and Well group, respectively, and "anxiety, stress or nerves" in $57.1 \%, 50 \%$ and $40 \%$, respectively. Fewer people used imidazopyrine for sleep ( $\mathrm{n}=7 \mathrm{CFS}, \mathrm{n}=6 \mathrm{ISF}, \mathrm{n}=2 \mathrm{Well}$ ), while, barbiturates were only occasionally used ( $n=2$ CFS and $n=1$ ISF) as ingredients of anti-migraine/headache drugs.

\section{Muscle relaxants}

Subjects in the CFS group used muscle relaxants significantly more frequently (9\%) than those in the ISF group (3\%) or Well controls ( $0 \%)$, see tables 2 and 3 .

\section{Hormones}

Persons with CFS were significantly more likely to use thyroid hormones only when compared to the ISF group $\left(\mathrm{OR}_{\text {adj. }}=2.60,95 \% \mathrm{CI}=1.03,6.57, \mathrm{p}=0.043\right)$ but not when compared to the Well group (table 3 ). In all groups the reported reason for thyroid hormone use was "hypothyroidism" or "thyroidectomy". Concurrent use of thyroid hormone and an antidepressant occurred in six persons from the CFS group (5.3\% of the whole group or $14.6 \%$ of persons with CFS who took antidepressants) and 4 from the ISF group (1.5\% of the entire ISF group or $9.8 \%$ of persons with ISF who took antidepressants) but in none from the Well group ( $\mathrm{p}$-value for linear trend = 0.004 , for the whole groups, $\mathrm{p}$-value for linear trend = 0.22 for the subgroups on antidepressants). However, no one reported use of thyroid hormones for the purpose of augmenting the effect of antidepressants.

The overall use of female hormone preparations among women was similar in the CFS (30.4\%) and Well (28\%) groups (Table 2). Despite the age-matching of CFS cases and Well controls, birth control drugs were used less frequently by the CFS group (6.5\% of females with CFS compared to $11.8 \%$ of the Well females and $9.9 \%$ of females with ISF) while hormone replacement use was greater among females with CFS (20.7\%) than in the ISF $(13.9 \%)$ or Well groups (14\%) but these differences did not reach statistical significance.

\section{Other drugs and supplements}

Compared to Well controls, CFS subjects used less frequently supplements and cardiovascular, lipid-lowering, and glucose-lowering drugs (tables 2 and 3). However, none of these differences reached statistical significance of 0.05 .

\section{Discussion}

In this cross-sectional, case-control study of CFS in Georgia we found that virtually all participants had used a drug or a supplement during the preceding two weeks $(95.6 \%$ of CFS, $88.6 \%$ of ISF, and $90.3 \%$ of Well controls). This is higher than the average estimate of $82 \%$ for the US popu- lation in 2004 and 2006 [16]. Among the three study groups, the highest prevalence of drug use occurred in the CFS group ( $93 \%$ used at least one drug), while the highest prevalence of supplement use occurred in the Well group ( 52.4\%).

Our findings confirm those from a previous study of medication use in persons with CFS from Wichita, Kansas [7]. Both studies found significantly higher usage of pain relievers, gastrointestinal drugs, antidepressants and benzodiazepines by persons with CFS compared to Well controls. Unlike the Wichita study, though, persons with CFS in Georgia were not significantly more likely than controls to use hormones and supplements but were significantly more likely than controls to use muscle relaxants and antiallergy and cold/sinus medications. Overall, compared to persons with CFS from the Wichita study [7], a smaller proportion of persons with CFS in Georgia used painrelievers (65.5\% in Georgia vs. 87.8\% in Wichita), supplements/vitamins $(44.3 \%$ vs. $62.2 \%)$, antidepressants ( $36.3 \%$ vs. $41.1 \%)$, antibiotics $(7.1 \%$ vs. $16.7 \%)$, hormones (43.4\% vs. $52.5 \%$. among women only, $11.8 \%$ among all CFS), antihypertensive drugs (17.7\% vs. $21.1 \%)$, muscle relaxants $(8.9 \%$ vs. $12.2 \%)$, anti-asthma medications $(7.1 \%$ vs. $12.2 \%)$, glucose-lowering drugs ( $0.9 \%$ vs. $4.4 \%$.). Use of other prescription drug categories such as lipid-lowering drugs (11.5\% vs.12.2\%) and benzodiazepines $(12.4 \%$, vs. $11.1 \%$ respectively) was similar in Georgia and Wichita (Kansas). The relatively lower usage of most prescription drug medications by persons with CFS in Georgia compared to Wichita may reflect lower seeking of, or lower access to, health care.

The more common use of pain-relievers by persons with CFS compared to those in the ISF and the Well groups is not surprising because joint and muscle aches belong to the symptom complex of CFS and because most painrelievers of the NSAID group are accessible over the counter. Persons with CFS used a variety of pain relieving/antiinflammatory drugs to treat arthritis and bodily pain, which predominated as reasons for NSAID use (in the CFS group). The significantly more common use of narcotic pain relievers by the CFS group, as compared to either the Well or the ISF groups, may be due to more severe pain and/or insufficient relief from conventional pain-relievers among persons with CFS. The $27 \%$ frequency of use of NSAIDs (aspirin excluded) among controls in our study appears comparable to the $32 \%$ estimated prevalence of joint pain in the general population of Georgia, or 33\% for the USA [17], as not all persons with joint/muscle pain take medications all the time. The different profile of NSAIDs use by the CFS and Well groups (i.e., ibuprofen was most commonly used by the CFS group and aspirin was most commonly used by the Well group), seems to reflect different reasons for use. Overall, almost 22\% of 
the Well controls used aspirin versus only $13 \%$ in the CFS and the ISF groups. Since the major reason for use of aspirin was "heart health"/prevention, it appears that more preventive use of aspirin occurred in the Well group. Use of acetaminophen-containing drugs in the CFS group ( $24 \%$ ) was higher than the estimated national average of $19 \%$, while both the Well controls and the ISF group had lower usage than the national average [16].

The higher frequency of antihistamine drugs most likely reflects higher prevalence of allergies and/or cold symptoms in the CFS population. It is notable also that the antihistamine use in our control group $(21.8 \%)$ was higher than the $15 \%$ antihistamine use in a control group of another U.S. study [18] and may reflect local practices and prescription patterns. In our study, about $20 \%$ of antihistamine users in the CFS and ISF groups used antihistamines as sleep aids, which was twice as much as that in the Well controls (11\%). Use of antihistamines, which have sleepiness and drowsiness as side effects, may also be an iatrogenic contribution to the CFS symptom complex.

Use of antidepressants by Well controls was $~ 9 \%$, mirroring the $9.2 \%$ national prevalence of depression over a 12 month period [19]. The more frequent use of psychotropic medications (antidepressants and sedatives) in the CFS group suggests that perhaps more depressed mood, anxiety and sleep disturbance are manifested by individuals fully meeting criteria for CFS. Indeed, in our study depression and anxiety were the most common psychiatric co-morbid conditions in persons with CFS [20]. Nevertheless, regardless of the more frequent use of antidepressants at higher mean dosages, persons with CFS and ISF had higher (worse) mean scores on the Zung selfrating depression scale than did Well controls. These results suggest that the clinical presentation of CFS, especially in subjects on antidepressants, may be related in part to untreated or treatment resistant symptoms of depression. Indeed, symptoms of fatigue in depressed patients have been found to be particularly resistant to conventional antidepressant therapy $[21,22]$. Moreover, depressed patients with early life stress - overrepresented in our CFS population [23], have also been shown to be less responsive to antidepressant medication [24]. Taken together, these results suggest that in some persons with CFS and depression, particularly those on antidepressants, unresolved depressive symptoms may significantly confound the diagnosis of CFS.

We were unable to find representative data for the use of acid-reducing drugs in the USA but the $12.9 \%$ use among the Well group was similar to the $10 \%$ overall use of antiacids (again within last two weeks) in other parts of the developed world [25]. Half of the of users of acid-reducing drugs in the CFS group also concurrently used
NSAIDs, whose major side effects are heartburn/acid reflux, gastritis, and even ulcers. At least one person from the CFS group specified that the reason for using anti-acid drugs was to counter side effects of an NSAID. Therefore, it is possible that anti-acids may have been used to treat side effects of NSAID drugs.

The $9 \%$ use of muscle relaxants in the CFS group was significantly greater not only when compared to the ISF (3\%) or the Well group (0\%) but also when compared to the national average of $1 \%$ [26]. In the national survey, half of the users of muscle relaxants took them for more than a year [26]. Because joint/muscle pain in CFS is chronic, persons in our study may also be taking muscle relaxants for extended periods of time and may experience their side effects (e.g., drowsiness, confusion, reduced alertness), which overlap with some of the CFS symptoms and may perpetuate them (i.e., iatrogenic effects of these drugs).

The approximately two-fold more common use of thyroid hormones in the CFS group compared to the ISF group deserves further study. Hypothyroidism presents a similar clinical picture to CFS; in fact, previously unrecognized hypothyroidism was the most common exclusionary condition detected during this study [8]. Autoimmune diseases are considered exclusionary for CFS as well, but were not particularly common in the study population [8]. Subjects who were successfully treated with thyroid replacement (as evidenced by TSH and T4 levels within the normal laboratory limits) were not excluded from our study. It is possible that some subjects treated with thyroid hormones may have chemically controlled hypothyroidism and CFS or, alternatively, they may be chemically euthyroid but functionally hypothyroid resulting in their presentation with CFS. Additional testing to address this possibility may be needed in future studies. Co-morbid depression and other psychiatric conditions were common in persons with CFS [20]. Thyroid hormones are sometimes prescribed to augment the effects of antidepressants [27] but there was no evidence for such indications in our study despite the combined use of thyroid hormone and an antidepressant by a few subjects in the CFS and the ISF group. Therefore, such use could not explain the higher frequency of thyroid hormone use by the CFS group in comparison to the ISF group.

Persons with CFS were taking, on average, approximately 6 preparations (ranging from 1 to 29 drugs and/or supplements). Polypharmacy (the use of multiple medications) raises the question of drug interactions, side effects and also the potential to use more drugs to treat symptoms that are side effects of drugs started earlier. The problem of iatrogenic symptoms is not trivial, particularly for chronic patients, as use of multiple drugs is an increasing problem 
[28]. The risks and consequences of polypharmacy should be a serious concern in the setting of CFS, where symptoms are chronic, treatment is largely only symptomatic, patients have about 22 doctors' visits per year [1] and may see multiple providers who independently prescribe different medications. Side effects of certain drugs may resemble symptoms of fatiguing illness. Therefore careful evaluation with respect to potential drug side effects and also drug-drug interactions is warranted for persons with CFS.

The findings from our study should be interpreted in view of its strengths and limitations. Major strengths of our study are its population-based design and the accuracy of the collected information: all drugs and supplements were brought to clinic where a research nurse viewed them and recorded the name and the dose. A limitation to consider is that reporting the reasons for drug/supplement use may not have been perfect, as subjects were not provided with a standardized list of reasons to choose from, and health literacy may have affected the accuracy of these data. Our study was cross-sectional in nature and does not allow for proper evaluation of treatment efficacy. Also, data on drug and supplement use limited to only two weeks may not be fully representative when studying a chronic, fluctuating condition such as CFS.

\section{Conclusion}

Our findings on medication use among persons with CFS, ISF and Well (controls) in Georgia have significant implications for both research and practice. Researchers should take into account that subjects with CFS usually take multiple drugs and supplements and that such use may be affecting study results (therefore, adjustments for or stratification by drug use may be needed in most studies of CFS). Future studies of drug and supplement use in subjects with CFS may need to be longitudinal, to focus on periods longer than two weeks, and collect additional data such as duration of treatment and source of prescription. Such studies may need to examine whether drug use is supported by underlying diagnoses. Also, more research is needed into the efficacy of antidepressant treatment in persons with CFS and whether it is related to history of early life stress. The most important implication for practice is that health care providers need to be aware of the use of multiple drugs and supplements (polypharmacy) in persons with CFS and consider the possible iatrogenic effects - both side effects from each drug and possible drug interactions - as potential contributors to the symptoms of their patients. Provider education programs for CFS may benefit from an overview of side effects of drugs more frequently used by persons with CFS.

\section{Competing interests}

The authors declare that they have no competing interests.

\section{Authors' contributions}

RSB cleaned, analyzed and interpreted the data, reviewed the literature and wrote the manuscript; JSL contributed to the statistical analysis; EMM and JFJ critically reviewed the manuscript and interpreted data; WCR was instrumental in the design of the population-based study and critically reviewed the manuscript. All authors read and approved the final version of the manuscript.

\section{Disclaimer}

The findings and views in this report are those of the authors and do not necessarily reflect the views of the funding agency.

\section{Additional material}

\section{Additional file 1}

Detailed version of table 3 - Adjusted odds ratios for associations between illness status and use of specific drug categories or supplements.

Click here for file

[http://www.biomedcentral.com/content/supplementary/14777525-7-67-S1.doc]

\section{Acknowledgements}

The authors acknowledge Daisy Lee, Elizabeth Unger, MD, of the CDC, Suzanne Vernon, PhD, formally of the CDC, and Christine Heim, $\mathrm{PhD}$, of Emory University, for their contributions to the study protocol; Andrew Miller, MD, of Emory University for his insightful comments; Marjorie Morrissey and Rebecca Devlin of Abt Associates for managing the study. The authors thank all the subjects who volunteered to participate in the study.

\section{References}

I. Bombardier $\mathrm{CH}$, Buchwald D: Chronic fatigue, chronic fatigue syndrome, and fibromyalgia. Disability and health-care use. Med Care 1996, 34:924-930.

2. Jason LA, Richman JA, Rademaker AW, Jordan KM, Plioplys AV, Taylor $R$, et al.: A community-based study of chronic fatigue syndrome. Archives of Internal Medicine 1999, 159:21 29-2137.

3. Reyes M, Nisenbaum R, Hoaglin DC, Unger ER, Emmons C, Randall $B$, et al.: Prevalence and incidence of chronic fatigue syndrome in Wichita, Kansas. Arch Intern Med 2003, 163:1530-1536.

4. Whiting P, Bagnall AM, Sowden AJ, Cornell JE, Mulrow CD, Ramirez $\mathrm{G}$ : Interventions for the treatment and management of Chronic fatigue Syndrome: a systematic review. JAMA 200I, 286: $1360-1368$.

5. Jason LA, Taylor RR, Kennedy CL, Song S, Johnson D, Torres S: Chronic fatigue syndrome: occupation, medical utilization, and subtypes in a community-based sample. J Nerv Ment Dis 2000, I 88:568-76.

6. Lin JM, Brimmer DJ, Boneva RS, Jones JF, Reeves WC: Barriers to healthcare utilization in fatiguing illness: a population-based study in Georgia. BMC Health Serv Res 2009, 9:I3.

7. Jones JF, Nisenbaum R, Reeves WC: Medication use by persons with chronic fatigue syndrome: results of a randomized telephone survey in Wichita, Kansas. Health Qual Life Outcomes 2003, I:74.

8. Reeves WC, Jones JF, Maloney E, Heim C, Hoaglin DC, Boneva RS, Morrissey M, Devlin R: Prevalence of chronic fatigue syndrome in metropolitan, urban, and rural Georgia. Popul Health Metr 2007, 5:. (doi: 10.1 186/1478-7954-5-5.)

9. Fukuda K, Straus SE, Hickie I, Sharpe MC, Dobbins JG, Komaroff A, International Chronic Fatigue Syndrome Study Group: The Chronic 
Fatigue Syndrome: A Comprehensive Approach to Its Definition and Study. Ann Intern Med 1994, I2 I(I 2953-959 [http:// www.annals.org/cgi/content/full//21//2/953].

10. Reeves WC, Lloyd A, Vernon SD, Klimas N, Jason LA, Bleijenberg G, Evengard $B$, White PD, Nisenbaum R, Unger ER, International Chronic Fatigue Syndrome Study Group: Identification of ambiguities in the 1994 chronic fatigue syndrome research case definition and recommendations for resolution. BMC Health Serv Res 2003, 3:25.

11. Zung WW: A self-rating depression scale. Arch Gen Psychiatry 1965, I 2:63-70.

12. Reeves WC, Wagner D, Nisenbaum R, Jones JF, Gurbaxani B, Solomon L, Papanicolaou DA, Unger ER, Vernon SD, Heim C: Chronic fatigue syndrome - a clinically empirical approach to its definition and study. BMC Med 2005, 3:19.

13. Ware JE, Sherbourne CD: The MOS 36-item short form health survey (SF-36): conceptual framework and item selection. Med Care 1992, 30:473-483.

14. Smets EM, Garssen B, Bonke B, De Haes JC: The Multidimensional Fatigue Inventory (MFI) psychometric qualities of an instrument to assess fatigue. J Psychosom Res 1995, 39:315-325.

15. Wagner D, Nisenbaum R, Heim C, Jones JF, Unger ER, Reeves WC: Psychometric properties of a symptom-based questionnaire for the assessment of chronic fatigue-syndrome. Popul Health Metr 2005, 3:8.

16. Sloan Survey, University of Boston [http://www.bu.edu/slone/ SloneSurvey/AnnualRpt/SloneSurveyWebReport2006.pdf]

17. CDC: Prevalence of self-reported arthritis or chronic joint symptoms among adults - United States, 200 I. MMWR Morb Mortal Wkly Rep 2002, 5 I (42948-950 [http://www.cdc.gov/mmwrl preview/mmwrhtml/mm5|42a2.htm].

18. Scheurer ME, El-Zein R, Thompson PA, Aldape KD, Levin VA, Gilbert MR, Weinberg JS, Bondy ML: Long-term Anti-inflammatory and Antihistamine Medication Use and Adult Glioma Risk. Cancer Epidemiol Biomarkers Prev 2008, I7(5):|277-I28I.

19. Marcotte DE, Wilcox-Gök V, Redmo DP: Prevalence and Patterns of Major Depressive Disorder in the United States Labor Force. J Ment Health Policy Econ 1999, 2(3): |23-|3|.

20. Nater UM, Lin JM, Maloney EM, Jones JF, Tian H, Boneva RS, Raison CL, Reeves WC, Heim C: Psychiatric Co-morbidity in Persons with Chronic Fatigue Syndrome Identified from the Georgia Population. Psychosom Med 2009, 7 I (5):557-565.

21. Fava M: Symptoms of fatigue and cognitive/executive dysfunction in major depressive disorder before and after antidepressant treatment. J Clin Psychiatry 2003, 64(Suppl I4):30-34.

22. Greco T, Eckert $G$, Kroenke $K$ : The outcome of physical symptoms with treatment of depression. Journal of General Internal Medicine 2004, | 9:8|3-8|8.

23. Heim C, Wagner D, Maloney E, Papanicolaou DA, Solomon L, Jones $J F$, Unger ER, Reeves WC: Early adverse experience and risk for chronic fatigue syndrome: results from a population-based study. Arch Gen Psychiatry 2006, 63:1258-66.

24. Nemeroff CB, Heim CM, Thase ME, Klein DN, Rush AJ, Schatzberg AF, Ninan PT, McCullough JP Jr, Weiss PM, Dunner DL, Rothbaum BO, Kornstein S, Keitner G, Keller MB: Differential responses to psychotherapy versus pharmacotherapy in patients with chronic forms of major depression and childhood trauma. Proc Natl Acad Sci USA 2003, 100: 14293-14296.

25. Furu K, Straume B: Use of antiacids in a general population: the impact of health-related variables, lifestyle and socio-economic characteristics. J Clin Epidemiol 1999, 52:509-5 I6.

26. Dillon C, Paulose-Ram R, Hirsch R, Gu Q: Skeletal muscle relaxant use in the United States: data from the Third National Health and Nutrition Examination Survey (NHANES III). Spine 2004, 29:892-6.

27. Carvalho AF, Cavalcante JL, Castelo MS, Lima MC: Augmentation strategies for treatment-resistant depression: a literature review. J Clin Pharm Ther 2007, 32:415-28.

28. Budnitz DS, Pollock DA, Weidenbach KN, Mendelsohn AB, Schroeder T, Annest JL: National surveillance of emergency department visits for outpatient adverse drug events. JAMA 2006, 296: 1858-1866

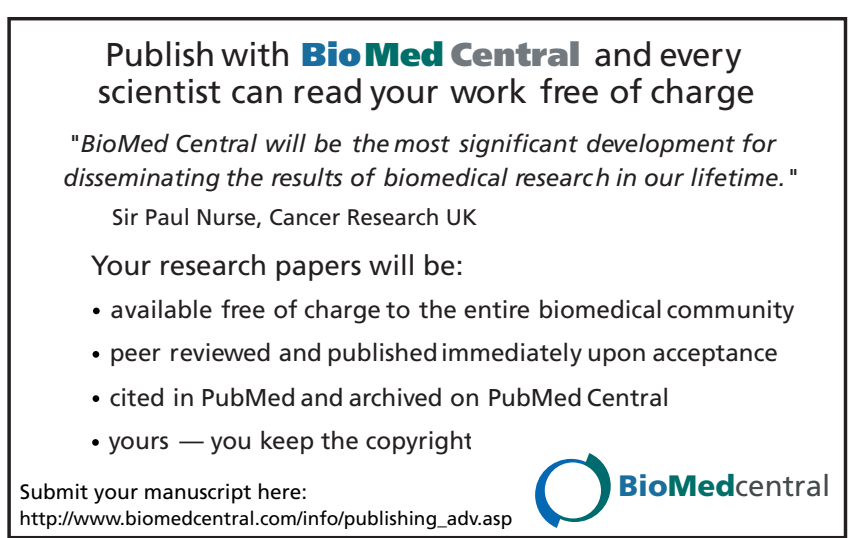

